\title{
Dímeros D e hiperglucemia y mortalidad en COVID-19 en un centro hospitalario de México
}

\author{
Carlos Martínez-Murillo ${ }^{1 *}$, Lourdes Basurto-Acevedo², Leticia Manuel-Apolinar², \\ Christian Ramos-Peñafiel11,3, Irma K. Pellón-Téllez', Beatriz Li-Gómez¹, Rodrigo Rivera-Tapia², \\ Estefanía Ledesma-Muñoz ${ }^{2}$, Gilberto Barranco-Lampón ${ }^{1}$ y Francisco Zazueta-Pozos ${ }^{1}$ \\ ${ }^{1}$ Servicio de Hematología, Hospital General de México Dr. Eduardo Liceaga, Ciudad de México; ${ }^{2}$ Hospital Regional de Alta Especialidad Ixtapaluca, \\ Ixtapaluca, Edo. Méx.; ${ }^{3}$ Unidad de Investigación Médica en Enfermedades Endocrinas, Centro Medico Nacional Siglo XXI, IMSS, Ciudad de México. \\ México
}

\section{Resumen}

Objetivo: Evaluar la asociación entre la concentración de glucosa al ingreso y los niveles circulantes de dímeros $D$ en pacientes hospitalizados por enfermedad por coronavirus 2019 (COVID-19). Pacientes y métodos: Se estudiaron 187 pacientes hospitalizados por COVID-19. Se evaluaron las características bioquímicas, la concentración de glucosa y dímeros $D$, la gravedad de la enfermedad definida por la presencia de neumonía y/o insuficiencia respiratoria que ameritó ventilación mecánica invasiva (VMI) y la causa del egreso hospitalario. Resultados: La edad promedio de los pacientes fue 52 años, el $68 \%$ eran hombres, un $40.8 \%$ con obesidad y un $23.5 \%$ con hipertensión. Del total de pacientes hospitalizados, el $45.5 \%$ presentaba diabetes o hiperglucemia a la admisión. La concentración de proteína $C$ reactiva y de dímeros $D(1,134[646.5-4,135.0]$ vs. 755 [548.7-1,780.0] ng/ml; $p=0.04]$ fue superior en pacientes con diabetes e hiperglucemia, en comparación con los pacientes con glucosa normal. Los pacientes que requirieron VMI presentaron también mayor concentración de dímeros $D$. Se observó una correlación directa entre las concentraciones de glucosa inicial y dímeros $D$ ( $r: 0.239 ; p=0.003$ ). Conclusión: En los pacientes con COVID-19 el estado hiperglucémico se asocia directamente con un incremento de la concentración de dímeros $D$. Los resultados de este estudio deben conducir a insistir en el control glucémico como estrategia fundamental en los pacientes con COVID-19.

PALABRAS CLAVE: Dímeros D. Hiperglucemia. Diabetes mellitus. COVID-19.

\section{D-dimers, hyperglycemia and mortality in COVID-19 in a hospital in Mexico}

\section{Abstract}

Objective: To evaluate the association between glucose level at admission and circulating levels of D-dimers in patients hospitalized for coronavirus disease 2019 (COVID-19). Methods: 187 patients hospitalized for COVID-19 were studied. Biochemical characteristics, glucose and D-dimers levels, severity of disease, defined by the presence of pneumonia and/or respiratory failure that required invasive mechanical ventilation (IVM) and the cause of hospital discharge were evaluated. Results: Age was 52 years, $68 \%$ were male, $40.8 \%$ with obesity and $23.5 \%$ with hypertension. Of the total of hospitalized patients, $45.5 \%$ had diabetes or hyperglycemia upon admission. Patients with diabetes and/or admission hyperglycemia had higher levels of protein C-reactive and D-dimers [(1134 (646.5-4135.0) vs. 755 (548.7-1780.0) $\mathrm{ng} / \mathrm{ml}, p=0.04]$, compared to patients with normal glucose level. Patients who required IMV also had a higher concentration of D-dimers. A correlation between glucose and $D$-dimers levels was evidenced ( $r=0.239, p=0.003)$. Conclusions: In patients with COVID-19, the hyper-

Correspondencia:

${ }^{*}$ Carlos Martínez-Murillo

E-mail: carlosmtzmurillo@gmail.com
Gac Med Mex. 2021;157(Supl 3):S10-S15

Disponible en PubMed

www.gacetamedicademexico.com

0016-3813/@ 2021 Academia Nacional de Medicina de México, A.C. Publicado por Permanyer. Este es un artículo open access bajo la licencia CC BY-NC-ND (http://creativecommons.org/licenses/by-nc-nd/4.0/). 
glycemic state is directly associated with an increase in the concentration of D-dimers and severity of disease. The results of this study should lead to insisting on glycemic control as a fundamental strategy in patients with COVID-19.

KEY WORDS: D-dimers. Diabetes mellitus. Hyperglycemia. COVID-19.

\section{Introducción}

A partir de la declaración de la Organización Mundial de la Salud de la enfermedad por el coronavirus-2019 (COVID-19) como pandemia, ha sido una cuestión importante identificar los factores de riesgo para infección severa y muerte. Dentro de los factores asociados se han señalado la edad superior a 65 años, el sexo masculino, la hipertensión, la obesidad y la diabetes mellitus (DM). En nuestro país, los datos han mostrado algunas diferencias, una de estas es la infección grave y mortalidad por COVID-19, que se presenta con mayor frecuencia en personas menores de 60 años, en contraste con otros países ${ }^{1}$.

En un estudio con información nacional extraída de la base de datos de la Dirección Nacional de Epidemiología de la Secretaría de Salud de México, en 51,633 pacientes confirmados como positivos para COVID-19 se encontró que el $18.3 \%$ padecía diabetes; este grupo de pacientes presentó además mayor riesgo de hospitalización, requerimiento de ventilación mecánica asistida y mortalidad². Otros estudios han mostrado una amplia variación de la frecuencia de diabetes, del 5.3 al $33.8 \%$, en pacientes con COVID-19 $9^{3,4}$.

Los mecanismos de asociación entre la diabetes y la infección por el coronavirus 2 del síndrome respiratorio agudo grave (SARS-CoV-2) no son claros. Se desconoce aún si la diabetes puede afectar la virulencia de la infección o si el virus altera el metabolismo de la glucosa ${ }^{5}$. Las personas con diabetes presentan más riesgo de infección por diferentes mecanismos, como son los defectos de la inmunidad innata que afectan la fagocitosis, la quimiotaxis de los neutrófilos y la inmunidad celular ${ }^{6}$. El estado proinflamatorio que se presenta en la diabetes favorece el proceso de tromboinflamación ${ }^{5}$. En los pacientes con diabetes se presenta un estado de hipercoagulabilidad, con aumento de la activación y agregación plaquetaria e incremento de diferentes factores de la coagulación como los FVIII, FXI y fibrinógeno 7 . Otro fenómeno que puede estar presente es la disminución de la fibrinólisis, debido a un incremento del inhibidor del activador del plasminógeno-1, y esta en relación con la resistencia a la insulina y al daño endotelial ${ }^{8}$.
El estado hipercoagulable que se asocia a COVID-19 se caracteriza por elevación de fibrinógeno y de los niveles de dímeros $\mathrm{D}$, así como otras alteraciones en el tiempo de protrombina, tromboplastina parcial activada y recuento plaquetario en los primeros estadios de la enfermedad. Los dímeros $D$ son productos de la escisión de la fibrina y su concentración elevada al ingreso hospitalario, así como su incremento durante la hospitalización, han servido como marcadores y se asocian con mal pronóstico y a fenómenos de microtrombosis ${ }^{9,10}$.

Por otra parte, la hiperglucemia detectada en el primer día de la hospitalización de los pacientes con COVID-19, independientemente de la preexistencia o no de diabetes, puede ser también un factor de mal pronóstico y se ha asociado con el hallazgo de alteraciones radiológicas de síndrome de dificultad respiratoria aguda en el paciente con COVID-911. La hiperglucemia, además de afectar a la respuesta inmunitaria, puede producir una respuesta inflamatoria anormal en los pacientes con COVID-1911,12.

En pacientes con COVID-19 existe poca información de la asociación entre la hiperglucemia y los niveles de dímeros $D$. El objetivo de este estudio fue evaluar la relación entre la glucosa circulante y los dímeros $\mathrm{D}$ en las primeras horas de hospitalización, así como su asociación con la gravedad de la enfermedad y riesgo de mortalidad.

\section{Material y métodos}

Se trata de un estudio observacional, retrospectivo, conducido en 187 pacientes hospitalizados con diagnóstico de COVID-19. El estudio se realizó en el Hospital General de México Eduardo Liceaga, el cual fue designado por el gobierno de México para la atención de pacientes con COVID-19. Los pacientes se atendieron en el periodo comprendido de marzo a junio de 2020.

Los criterios de inclusión fueron edad de 18 a 80 años y confirmación de COVID-19 por un examen positivo para SARS-CoV2 mediante reacción en cadena de la polimerasa en tiempo real, certificado por el Instituto Nacional de Diagnóstico y Epidemiología. Se revisaron los expedientes los pacientes y se 
obtuvo información sobre los antecedentes clínicos, los signos vitales y estudios de laboratorio obtenidos de los registros médicos. Se capturaron datos como edad, sexo, morbilidad e índice de masa corporal (IMC). Los datos de laboratorio recogidos fueron biometría hemática, glucosa sérica, química sanguínea, función hepática, fibrinógeno y dímeros $D$.

Se comparó la información de pacientes con COVID-19 y diabetes preexistente y/o hiperglucemia a su ingreso hospitalario, con la información de los pacientes con COVID-19 sin diabetes y con nivel de glucosa normal a su ingreso hospitalario. Se confirmó el diagnóstico de diabetes mediante el análisis de los registros médicos. Se definió la hiperglucemia al ingreso como glucosa sérica $\geq 140 \mathrm{mg} / \mathrm{dl}$ en las primeras 24 horas posteriores a la admisión hospitalaria. El valor incluido de la glucosa sérica fue en todos los casos anterior a la administración de glucocorticoides. Se capturó también información de los pacientes que requirieron ventilación mecánica invasiva (VMI) y la fecha de egreso hospitalario o fallecimiento.

\section{Análisis estadístico}

Para determinar el tipo de distribución de las variables se aplicó la prueba de Kolmogorov-Smirnov. Se calcularon las medidas de tendencia central y dispersión para valores no paramétricos, esto es, mediana y rangos intercuartílicos (p25-p75). Para comparar los grupos se utilizó la prueba $U$ de Mann-Whitney. Las diferencias entre las variables cualitativas se establecieron con la prueba $\chi^{2}$ y el análisis de correlación con el coeficiente de Spearman. Se consideró valor significativo estadísticamente cuando $p \leq 0.05$. El análisis estadístico se efectuó con el programa estadístico Statistical Package for the Social Sciences (SPSS) v20.

\section{Resultados}

Se incluyeron en el estudio 187 pacientes que cumplieron con los criterios de selección. La edad promedio de los pacientes fue 52 años, el 31.5\% fueron mujeres y el $68.5 \%$ hombres. Del total de pacientes, el $36.2 \%$ tenían diagnóstico de diabetes y ascendía al $45.5 \%$ cuando se consideró adicionalmente la hiperglucemia detectada al ingreso. El $23.5 \%$ de los participantes presentaban hipertensión arterial y el $40.8 \%$, obesidad.

En la tabla 1 se presentan las características de los pacientes sin diabetes y con diabetes e hiperglucemia a la admisión. No se observaron diferencias significativas en el IMC y frecuencia de obesidad entre los grupos. La concentración de proteína $C$ reactiva fue superior en los pacientes con diabetes e hiperglucemia a la admisión. En contraste, este último grupo presentó menor recuento linfocitario y saturación de oxígeno. El nivel de fibrinógeno fue similar en ambos grupos, mientras que la concentración de dímeros $D$ fue superior en el grupo de pacientes con diabetes e hiperglucemia a la admisión, en comparación con los pacientes $\sin$ diabetes $(1,134[646.5-4,135.0] \quad$ vs. 755 $[548.7-1,780.0] \mathrm{ng} / \mathrm{ml} ; \mathrm{p}=0.04)$. Además, los pacientes con diabetes e hiperglucemia requirieron más frecuentemente VMI, en comparación con los pacientes no diabéticos (64.7 vs. $27.8 \% ; p=0.001$ ).

Los pacientes que requirieron VMI por gravedad de COVID-19, en comparación con el grupo de pacientes con menos gravedad, presentaron incremento de la concentración de glucosa (137 [105.5229.7 vs. 110 [92-147] mg/dl; $p=0.003)$, y de dímeros D $(1,433[700-3,750]$ vs. $866[560.5-2,159] \mathrm{ng} / \mathrm{dl}$; $p=0.02$ ). De la misma forma, se observó que los pacientes que fallecieron presentaron mayor concentración de glucosa al ingreso a hospitalización (134 [108.5-206.5] vs. 117 [ 94-177.2] mg/dl; $p=0.05)$, así como del nivel de dímeros $D(1,580[1,024.5-4,234]$ vs. 1,000 [ $610-2,623] \mathrm{ng} / \mathrm{ml} ; \mathrm{p}=0.05)$, en comparación con los sobrevivientes. El nivel de dímeros $D$ no mostró diferencias significativas entre los pacientes con y sin hipertensión arterial (902 [560.5-3,104] vs. 860 [700-2,550.5] ng/ml, NS).

Se observó una correlación directa entre las concentraciones de glucosa y los dímeros D (Fig. 1). La glucemia correlacionó también con la edad ( $\mathrm{r}: 0.148$, $p=0.02)$ y con la proteína $C$ reactiva ( $r$ : 0.239 , $p=0.003$ ), pero no se asoció al IMC. De la misma forma, los dímeros $\mathrm{D}$ correlacionaron con la proteína $C$ reactiva ( $r: 0.147, p=0.05)$.

\section{Discusión}

En el presente trabajo se encontró una asociación directa entre la hiperglucemia de la admisión a hospitalización y la concentración de dímeros $\mathrm{D}$, la cual se ha demostrado que es un marcador de mortalidad para COVID-19.

La DM y otras comorbilidades como la obesidad y la hipertensión arterial son factores de riesgo de severidad y mortalidad para las infecciones producidas por los virus SARS y SARS-CoV-2 $2^{13,14}$. En nuestro estudio se encontró una alta frecuencia de pacientes 
Tabla 1. Características de los participantes de acuerdo con la presencia o no de diabetes mellitus

\begin{tabular}{|c|c|c|c|c|}
\hline Variable & $\begin{array}{c}\text { Total } \\
(n=187)\end{array}$ & $\begin{array}{l}\text { Sin diabetes } \\
(n=102)\end{array}$ & Diabetes e hiperglucemia $(n=85)$ & Valor $p^{*}$ \\
\hline Edad (años) & $52.0(42.2-60.0)$ & $51(40-59)$ & $52.2(42-60)$ & NS \\
\hline $\begin{array}{l}\text { Sexo } \\
\quad \text { Mujeres (\%) } \\
\text { Hombres (\%) }\end{array}$ & $\begin{array}{l}31.5 \\
68.5\end{array}$ & $\begin{array}{l}31.9 \\
68.1\end{array}$ & $\begin{array}{l}31.1 \\
68.9\end{array}$ & NS \\
\hline IMC $\left(\mathrm{kg} / \mathrm{m}^{2}\right)$ & $27.7(25.4-32.4)$ & $27.4(24.9-32.5)$ & $28.5(25.4-31.9)$ & NS \\
\hline Obesidad (\%) & 40.8 & 38.9 & 42.9 & NS \\
\hline Hipertensión (\%) & 23.5 & 19.6 & 28.8 & 0.01 \\
\hline Glucosa (mg/dl) & $118(94-175)$ & $103(86-118.5)$ & $209.5(134-314.2)$ & 0.0001 \\
\hline Urea (mg/dl) & $29.8(16.8-50.0)$ & $24.9(14.9-48.2)$ & $35(19.1-56.0)$ & 0.001 \\
\hline Creatinina (mg/dl) & $0.95(0.8-2.0)$ & $0.90(0.7-1.4)$ & $1.0(0.7-2.1)$ & NS \\
\hline Saturación de oxígeno (\%) & $89.0(78.1-93.0)$ & $89.1(86.0-92.4)$ & $87(70.4-91.9)$ & 0.05 \\
\hline Proteína C reactiva (mg/l) & $63.0(23.8-163.0)$ & $19.5(5.7-32.5)$ & $84.9(23.1-273.0)$ & NS \\
\hline Cuenta de neutrófilos (x109/I) & $7.88(5.0-11.1)$ & $7.22(5.24-10.53)$ & $8.1(5.7-11.5)$ & NS \\
\hline Linfocitos (x109/l) & $0.90(0.60-1.8)$ & $0.93(0.63-1.19)$ & $0.74(0.61-1.06)$ & 0.05 \\
\hline Plaquetas $\left(\times 10^{9} / 1\right)$ & $2421(189-330)$ & 252 (183-338.2) & $237.5(191-322.5)$ & NS \\
\hline Fibrinógeno (mg/dl) & $611(505-772)$ & $608.0(539.5-797.5)$ & $619.5(502.2-809.7)$ & NS \\
\hline Dímeros D (ng/ml) & $914(548-3,338)$ & $755.0(548.7-1,780.0)$ & $1,134(646.5-4,135.0)$ & 0.04 \\
\hline VMI (\%) & 40.8 & 27.8 & 64.7 & 0.001 \\
\hline
\end{tabular}

*Se compara el grupo con diabetes vs. el grupo sin diabetes. Las variables se presentan como mediana y rango intercuartílico. IMC: índice de masa corporal; VMI: ventilación mecánica invasiva; NS: no significativo.

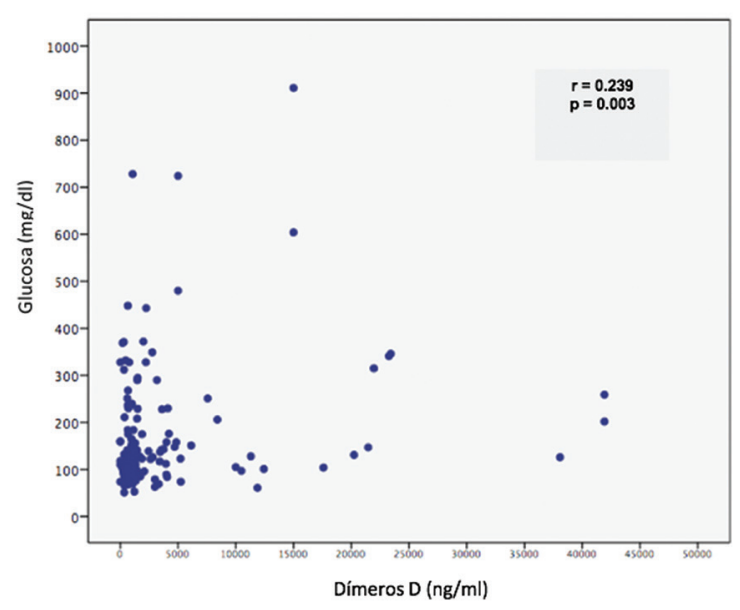

Figura 1. Se presenta la correlación entre la concentración de glucosa inicial en pacientes hospitalizados por enfermedad por coronavirus 2019 y la concentración de dímeros $D$.

con diagnóstico previo de diabetes $(36 \%)$ y llamó la atención el incremento de la frecuencia al $45.5 \%$ cuando se consideró adicionalmente la hiperglucemia detectada al ingreso de la hospitalización. Esta cifra por sí misma es alarmante.

Algunos de los mecanismos propuestos para explicar el efecto negativo de la hiperglucemia en la evolución de COVID-19 son las alteraciones en diferentes componentes de la respuesta inmunitaria del huésped, el estado inflamatorio y la desregulación de citocinas, que disminuyen la capacidad para combatir la infección del paciente con diabetes, lo que conlleva eventualmente un desenlace fatal14,15. Otro posible mecanismo es el efecto directo del SARS-CoV-2 sobre las células $\beta$ del islote pancreático. La infección por este virus podría conducir al deterioro directo de las células del islote pancreático y disminución de la secreción de insulina, resultando en el desarrollo de diabetes de novo ${ }^{15,16}$. En sentido inverso, la hiperglucemia en el islote pancreático favorece la producción de interleucina $1 \beta$, amplificando el proceso inflamatorio ${ }^{17}$.

En el presente trabajo se consideró no solo a pacientes con diabetes preexistente, sino también con 
hiperglucemia detectada al ingreso. La hiperglucemia detectada por vez primera en COVID-19 puede obedecer a una diabetes de reciente inicio, a hiperglucemia asociada a estrés, o inducida por efecto directo del SARS-CoV-2 ${ }^{4,6}$.

Estudios previos en pacientes con COVID-19 que desarrollaron síndrome respiratorio agudo severo han mostrado que la diabetes y la hiperglucemia durante el curso temprano de la enfermedad son predictores independientes de la mortalidad y morbilidad ${ }^{14,15}$. Otra observación ha sido que los pacientes hospitalizados con COVID-19 y diabetes recién diagnosticada presentaban mayor riesgo de mortalidad en comparación con los pacientes con diabetes previamente conocida o con glucosa norma ${ }^{12}$.

lacobellis, et al. encontraron que la hiperglucemia medida a la admisión hospitalaria fue el mejor predictor de imágenes radiográficas de COVID-19 sugestivas de gravedad, independientemente del historial previo de diabetes ${ }^{11}$. Los autores de ese trabajo propusieron que la hiperglucemia aguda puede conducir a una respuesta inflamatoria e inmunitaria anormal que contribuye al desarrollo y la progresión de la COVID-19'11.

En un estudio con los datos de la Dirección General de Epidemiología de la Secretaría de Salud de México, se informó que los pacientes con COVID-19 y diabetes de inicio temprano presentaban mayor riesgo de hospitalización y de mortalidad, así como en pacientes jóvenes y de mayor edad con diabetes ${ }^{2}$. La coexistencia de obesidad y diabetes, particularmente de inicio reciente, constituyen un factor de riesgo de mortalidad por COVID19 en los mexicanos ${ }^{2,18}$.

La hiperglucemia, además de su relación con el proceso inflamatorio e inmunitario, tiene un papel importante en la coagulopatía del paciente diabético. En la DM se presentan alteración de la sensibilidad a la insulina y mecanismos de glucosilación avanzada, que pueden activar algunas proteínas de la coagulación como el fibrinógeno, FVII y FvW, y antitrombina. A lo anterior se sumaría el daño endotelial producido por el virus, todo lo que conduce a elevación de dímeros $\mathrm{D}^{7}$.

Un dato relevante del presente estudio fue la asociación directa entre la hiperglucemia y los dímeros D. Este resultado sugiere que la elevación de la glucosa en los pacientes con COVID-19 no solo conduce a una respuesta inflamatoria, sino también a alteración del sistema de coagulación.
Los resultados del presente estudio coinciden con un estudio reciente, en el que se encontró que la hiperglucemia originaba un estado inflamatorio y alteraciones del sistema coagulación, que favorecen la gravedad y mortalidad en la COVID-19 ${ }^{16,19}$.

Los dímeros $D$ se originan de la formación y lisis de fibrina y son un reflejo de la activación de la coagulación y de la fibrinólisis9. La concentración de dímeros $D$ es un predictor independiente de la mortalidad hospitalaria en pacientes con COVID-199,11. Se ha planteado que el estado hipercoagulable en COVID-19 está condicionado por una respuesta proinflamatoria intensa, disfunción endotelial, condiciones de protrombosis y coagulopatía inducida por el estado séptico, y puede evolucionar a una coagulación intravascular diseminada ${ }^{19,20}$.

Las limitaciones del presente estudio incluyen el diseño retrospectivo y su realización en un centro hospitalario, estudiando pacientes con COVID-19 con estado de gravedad, por lo que los resultados de este estudio no necesariamente se pueden generalizar a pacientes con cuadros de COVID-19 de menor intensidad. Otra debilidad del estudio es la falta de recursos para medición de la hemoglobina glucosilada, que hubiera permitido definir a pacientes con hiperglucemia aguda y crónica. Una fortaleza del estudio es precisamente su realización en un entorno clínico, en donde se evaluaron pacientes con cuadro de COVID-19 con mayor intensidad; en este grupo de pacientes se debe identificar los mayores riesgos por su riesgo de evolucionar a un estado de mayor gravedad y mortalidad.

El estudio de la asociación entre hiperglucemia y alteraciones de la coagulación, así como de su relación con cuadros severas de COVID-19, debe extenderse, particularmente en un país como México, que presenta una elevada prevalencia de diabetes y mortalidad por infección por SARS-CoV-2.

\section{Conclusión}

En los pacientes con COVID-19, el estado hiperglucémico se asocia directamente con un incremento de la concentración de dímeros D. La hiperglucemia favorece mecanismos proinflamatorios y protrombóticos, sin embargo, se requiere ampliar los estudios sobre la relación de la hiperglucemia con la fibrinólisis en el paciente con COVID-19.

Los resultados del presente estudio deben conducir a insistir en el control glucémico como estrategia fundamental en los pacientes que presentan COVID-19. 


\section{Conflictos de intereses}

Los autores manifiestan no tener conflicto de intereses alguno.

\section{Financiamiento}

La presente investigación no ha recibido financiamiento ni patrocinio por ninguna organización 0 institución.

\section{Responsabilidades éticas}

Protección de personas y animales. Los autores declaran que para esta investigación no se han realizado experimentos en seres humanos ni en animales.

Confidencialidad de los datos. Los autores declaran que en este artículo no aparecen datos de pacientes.

Derecho a la privacidad y consentimiento informado. Los autores declaran que en este artículo no aparecen datos de pacientes.

\section{Bibliografía}

1. Rod JE, Trespalacios O, Cortes J. A brief-review of the risk factors for covid-19 severity. Rev Saúde Pública. 2020;54:60-8.

2. Bello-Chavolla O, Bahena-López P, Villa N, Vargas-Vázquez A, González-Díaz A, Márquez-Salinas A, et al. Predicting mortality attributable to SARS-CoV-2: A mechanistic score relating obesity and diabetes to $\mathrm{CO}$ VID-19 outcomes in Mexico. J Clin Endocrinol Metab. 2020;105(8):1-10.

3. Richardson S, Hirsch JS, Narasimhan M, Crawford M, McGinn T, Davidson W, et al. Presenting characteristics, comorbidities, and outcomes among 5700 patients hospitalized with COVID-19 in the New York City area. JAMA. 2020;323:2052-9.
4. Hussain A, Bhowmik B, Moreira N. COVID-19 and diabetes: Knowledge in progress. Diabetes Res Clin Pract. 2020;162:108-42.

5. Bornstein SR, Rubino F, Khunti K, Mingrone G, Hopkins D, Birkenfeld $A L$. Practical recommendations for the management of diabetes in patients with COVID-19. Lancet Diabetes Endocrinol. 2020;8:547-50.

6. Van der Toorn FA, de Mutsert R, Lijfering WM, Rosendaal FR, van Hylckama Vlieg A. Glucose metabolism affects coagulation factors: The NEO study. J Thromb Haemost. 2019;17:1886-97.

7. Burton ES, Schneider DJ. Platelet function, coagulopathy, and impaired fibrinolysis in diabetes. Cardiol Clin. 2004;22:511-26.

8. Gupta A, MadhavanV, Sehgal K, Nair N, MahajanS, Sehrawat T, et al. Extrapulmonary manifestations of COVID-19. Nat Med. 2020;26:1017-32.

9. Ye W, Chen G, Li X, Lan X, Chen J, Min H, et al. Dynamic changes of D-dimer and neutrophil-lymphocyte count ratio as prognostic biomarkers in COVID-19. Respiratory Res. 2020;21:169.

10. lacobellis G, Malavazos AE, Ferreira T. COVID-19 rise in younger adults with obesity: Visceral adiposity can predict the risk. Obesity (Silver Spring). 2020;28(10):1795.

11. lacobellis G, Penaherrera C, Bermudez L, Bernal E. Admission hyperglycemia and radiological findings of SARS-CoV2 in patients with and without diabetes. Diabetes Res Clin Pract. 2020;164:108185.

12. Li X, Xu S, Yu M, Wang K, Tao Y, Zhou Y, et al. Risk factors for severity and mortality in adult COVID-19 inpatients in Wuhan. J Allergy Clin Immunol. 2020;146:110-8.

13. Maddaloni E, Buzzetti R. Covid-19 and diabetes mellitus: unveiling the interaction of two pandemics. Diabetes Metab Res Rev. 2020 Mar 31;e33213321. doi: 10.1002/dmrr.3321. Online ahead of print.

14. Yang JK, Feng Y, Yuan MY, Yuan SY, Fu HJ, Wu B, et al. Plasma glucose levels and diabetes are independent predictors for mortality and morbidity in patients with SARS. Diabet Med. 2006;23:623-8.

15. Cuschieri S, Grech S. COVID-19 and diabetes: The why, the what and the how. J Diabetes Complications. 2020;34(9):107637.

16. Apicella M, Campopiano MC, Mantuano M, Mazoni L, Cappelli A, Del Prato S. COVID-19 in people with diabetes: understanding the reasons oir worse outcomes. Lancet Diabetes Endocrinol. 2020;8(9):782-92.

17. Tsalamandris S, Antonopoulos AS, Oikonomou E, Papamikroulis G, Vogiatzi G, Papaioannou S, et al. The role of inflammation in Diabetes: Current concepts and future perspectives. Eur Cardiol. 2019;14:50-9.

18. Denova-Gutierrez E, López-Gatell H, Alomia-Zegarra JL, López-Ridaura R, Zaragoza-Jiménez CA, Dyer-Leal DD, et al. The association of obesity, type 2 diabetes, and hypertension with severe Coronavirus disease 2019 on admission among Mexican patients. Obesity (Silver-Spring). 2020;28(10):1826-32.

19. Bode B, Garrett V, Messler J, McFarland R, Crowe J, Booth R, et al. Glycemic characteristics and clinical outcomes of COVID-19 patients hospitalized in the United States. J Diabetes Sci Technol. 2020;14:813-21.

20. Pérez-Martínez P, Carrasco Sánchez FJ, Carretero Gómez J, Gomez-Huelgas R. Resolviendo una de las piezas del puzle: COVID-19 y diabetes tipo 2. Rev Clin Esp. 2020;220(8):507-10. 